\title{
Effective equations for two-phase flow in porous media: the effect of trapping on the microscale
}

\author{
Cornelis Johannes van Duijn • Hartmut Eichel • \\ Rainer Helmig • Iuliu Sorin Pop
}

Received: 27 September 2005 / Accepted: 4 November 2006 / Published online: 25 January 2007

(C) Springer Science+Business Media B.V. 2007

\begin{abstract}
In this article, we consider a two-phase flow model in a heterogeneous porous column. The medium consists of many homogeneous layers that are perpendicular to the flow direction and have a periodic structure resulting in a one-dimensional flow. Trapping may occur at the interface between a coarse and a fine layer. Assuming that capillary effects caused by the surface tension are in balance with the viscous effects, we apply the homogenization approach to derive an effective (upscaled) model. Numerical experiments show a good agreement between the effective solution and the averaged solution taking into account the detailed microstructure.
\end{abstract}

Keywords Homogenization · Two-phase flow · Porous media · Upscaling

\section{Introduction}

Two-phase flow processes in heterogeneous porous media occur in various natural systems. For example, when considering groundwater remediation, or oil recovery from reservoirs. Usually these natural systems display heterogeneities that range from small-scale inclusions or lenses to large-scale formations. Two different homogeneous materials are often separated by a sharp interface, the transition from one material to another being non-smooth. A significant amount of research has been undertaken to quantify the effects of such transitions on the movement of the phases. One of the first qualitative studies of the effects of heterogeneities on two-phase porous media flow has been done by Kueper et al. (1989). Laboratory experiments using a porous column with periodically varying permeability were performed by van Lingen (1998).

C. J. van Duijn · I. S. Pop

Department of Mathematics and Computer Science,

TU Eindhoven, PO Box 513, 5600 MB Eindhoven, The Netherlands

H. Eichel $(\varangle) \cdot$ R. Helmig

Department of Hydromechanics and Modeling of Hydrosystems, Institute of Hydraulic Engineering, Universität Stuttgart, Pfaffenwaldring 61, 70550 Stuttgart, Germany

e-mail: hartmut.eichel@iws.uni-stuttgart.de 
In the case of small-scale heterogeneities, the geological information is hardly ever available. Moreover, even if complete information could be obtained, the computational effort required to perform complete simulations on the microscale is immense. Therefore, it is necessary to obtain effective equations, parameters or constitutive relationships, being able to capture the effects of smallscale heterogeneities and transfer them to our modeling scale. There exists a variety of methods to obtain effective or upscaled descriptions. We refer to Wen and Gómez-Hernández (1996) and Darman et al. (2002) for overviews.

In this article, we employ homogenization techniques to describe the effect of microscale heterogeneities. The essence of this approach, together with many examples, is explained in Hornung (1997). Homogenization techniques have been successfully applied by Neuweiler and Cirpka (2005) and Lewandowska et al. (2004) to upscale flows in unsaturated porous media. This article is a continuation of van Duijn et al. (2002a), where two-phase flow with trapping at the microscale is considered. In this article, we consider the capillary limit case of van Duijn et al. (2002a), in which the capillary effects caused by the surface tension are in balance with viscous effects. In the dimensionless setting this is expressed by a capillary number of order one. In the absence of a rigorous mathematical proof for the convergence of the homogenization procedure, the results in van Duijn et al. (2002a) are obtained assuming that the first-order term in the asymptotic expansion of the oil saturation is continuous across the interfaces separating the homogeneous layers. This leads to enhanced diffusive effects in the resulting effective equation. In this article, we assume the continuity of the first-order term in the asymptotic expansion of the capillary pressure, thus giving up the continuity of the corresponding term for the oil saturation. Even though these assumptions refer to terms of small order, they lead to different macroscale results, as we will see below.

In Sect. 2 we present the model and give the governing equations and interface conditions. Further, the model is transformed into a dimensionless setting in which the capillary number appears. In Sect. 3 we briefly recall the homogenization approach and derive the effective equations incorporating the microscale interface conditions. The results are discussed in Sect. 3.2. Numerical simulations are performed in Sect. 4. There we compare the solution of the effective equation to the result of simulations of the microscale, i.e., multilayered flow process. The article concludes with some final remarks.

\section{The model}

In this section, we describe the specific two-phase flow model and the conditions at the interfaces separating two different homogeneous media.

\subsection{Model: set-up, equations and assumptions}

The medium is assumed to consist of periodically repeating homogeneous layers that are perpendicular to the flow direction. This results in a one-dimensional flow and a spatially periodic medium consisting of alternating coarse and fine layers. For the simplicity of presentation we assume here that the layers have a constant porosity $\Phi$, but a variable absolute permeability $k=k(x)$ that is assumed constant inside each of these layers. Therefore we investigate the one-dimensional flow of two immiscible 


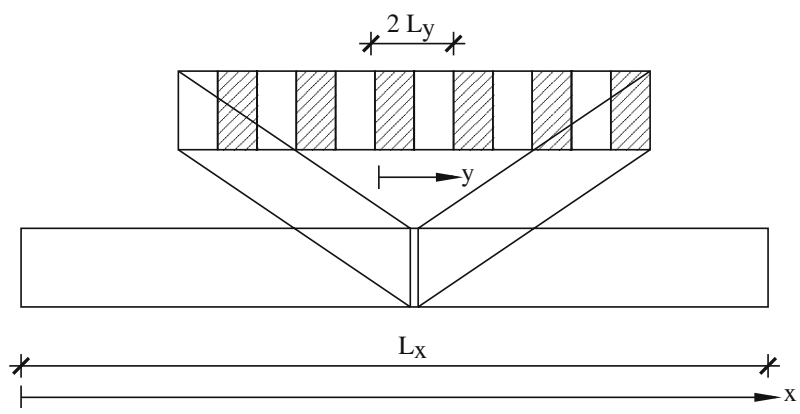

Fig. 1 Small scale and large scale

and incompressible phases (water being the wetting phase, oil the non-wetting phase) through a periodic medium with alternating homogeneous high and low permeability zones. The ratio of the (microscale) homogeneous layer thickness $L_{y}$ and the (macroscale) column length $L_{x}$ is assumed small-see Fig. 1.

The governing equations are the mass conservation:

$$
\phi \frac{\partial S_{\alpha}}{\partial t}+\frac{\partial q_{\alpha}}{\partial x}=0 \quad(\text { with } \alpha=\mathrm{w}, \mathrm{nw}),
$$

and the extended Darcy law:

$$
q_{\alpha}=-k(x) \frac{k_{r \alpha}\left(S_{\alpha}\right)}{\mu_{\alpha}} \frac{\partial p_{\alpha}}{\partial x} .
$$

The system is closed with the complementary conditions:

$$
\begin{aligned}
& S_{\mathrm{nw}}+S_{\mathrm{w}}=1, \\
& p_{\mathrm{nw}}-p_{\mathrm{w}}=p_{\mathrm{c}}\left(x, S_{\mathrm{w}}\right) .
\end{aligned}
$$

Here $\phi, S, \mu, p$, and $q$ denote the porosity, the saturation-mapped to the standard interval $[0,1]$, the viscosity, the phase pressure and the specific discharge, respectively, with subscripts for each of the phases. $k$ denotes the intrinsic (absolute) permeability, and $k_{\mathrm{r}}$ is the relative permeability of the respective phase. The capillary pressure is described by the Leverett model (Leverett, 1941):

$$
p_{\mathrm{c}}\left(x, S_{\mathrm{W}}\right)=\sigma \sqrt{\frac{\phi}{k(x)}} J\left(S_{\mathrm{W}}\right) .
$$

where $\sigma$ and $J$ are the interfacial tension between the two fluids and the Leverett function, respectively. Here we assume that $J$ is strictly decreasing, going to infinity as $S_{\mathrm{W}} \searrow 0$, and that $J(1)>0$. Physically this means that the pressure difference in the two phases should exceed the capillary entry pressure given by $p_{\mathrm{c}}(x, 1)=\sigma \sqrt{\frac{\phi}{k(x)}} J(1)$, before the oil can enter into a fully water saturated medium. We immediately see that the entry pressure is inversely proportional to the square root of the permeability, and therefore varies between the layers. Equation 1 and condition (3) imply that the total specific discharge $q:=q_{\mathrm{w}}+q_{\mathrm{nw}}$ is constant in space. Throughout this article we consider it constant in time as well. Therefore we assume $q>0$ being a given quantity, meaning that the flow takes place from left to right. Having this in mind we 
can combine Eqs. 1, 2 and conditions (3), (4) into a single transport equation for the oil saturation:

$$
\phi \frac{\partial S_{\mathrm{nw}}}{\partial t}+\frac{\partial}{\partial x}\left[\frac{k_{\mathrm{nw}}}{k_{\mathrm{nw}}+k_{\mathrm{w}} \mu_{\mathrm{o}} / \mu_{\mathrm{w}}} q-\frac{k(x)}{\mu_{\mathrm{w}}} \frac{k_{\mathrm{nw}} k_{\mathrm{w}}}{k_{\mathrm{nw}}+k_{\mathrm{w}} \mu_{\mathrm{o}} / \mu_{\mathrm{w}}} \frac{\partial p_{\mathrm{c}}}{\partial x}\right]=0
$$

For being consistent with van Duijn et al. (2002a), we set

$$
u=S_{\mathrm{nw}} \quad\left(S_{\mathrm{w}}=1-u\right)
$$

and redefine $k_{\mathrm{rw}}, p_{\mathrm{c}}$, and $J$ in terms of $u$. In this way $J$ becomes strictly increasing in $u$, goes to infinity as $u \nearrow 1$ and $J(0)>0$. With $L_{x}$ being a characteristic macroscopic length scale and $K$ a characteristic permeability, we apply the scalings:

$$
\hat{x}:=\frac{x}{L_{x}}, \quad \hat{t}:=\frac{t q}{\phi L_{x}}, \quad \hat{k}:=\frac{k}{K},
$$

the hats indicating dimensionless variables. However, in what follows we give up the hats, all the variables and unknowns having no dimensions. We do this to simplify the notation. In this way we bring (6) to a dimensionless form:

$$
\frac{\partial u}{\partial t}+\frac{\partial F}{\partial x}=0
$$

with

$$
F=f(u)-N_{\mathrm{c}} k(x) k_{\mathrm{rw}}(u) f(u) \frac{\partial}{\partial x} p_{\mathrm{c}}(x, u) .
$$

Here

$$
f(u)=\frac{k_{\mathrm{ro}}(u)}{k_{\mathrm{ro}}(u)+M k_{\mathrm{rw}}(u)}
$$

is the fractional flow function, and

$$
p_{\mathrm{c}}(x, u)=\frac{J(u)}{\sqrt{k(x)}}
$$

is the capillary pressure. The capillary number $N_{\mathrm{c}}$ and the viscosity ratio $M$ from above are defined as follows

$$
N_{\mathrm{c}}=\frac{\sigma \sqrt{K \phi}}{\mu_{\mathrm{w}} q L_{x}}, \quad M=\frac{\mu_{\mathrm{o}}}{\mu_{\mathrm{w}}} .
$$

Depending on the specific application, the value of the capillary number $N_{\mathrm{c}}$ may vary considerably. In van Duijn et al. (2002a), the consequences of having a moderate and a small value for $N_{\mathrm{c}}$ are analyzed separately, resulting into different types of effective equations. Here we concentrate on the case of moderate $N_{\mathrm{c}}$.

\subsection{The entry pressure effect}

Physical experiments (Illangasekare et al. 1995; Kueper et al. 1989) have shown that the capillary forces have an important influence on the two-phase flow behavior in porous media. This impact is easy to recognize in heterogeneous soils, especially at the interfaces between different materials. The so-called entry pressure effect, i.e., the inability of a non-wetting fluid to invade a fine grained material, has been observed 
in the aforementioned experiments and was thereafter also discussed and modeled in a couple of papers (Helmig 1997; Kueper and Frind 1991).

The entry pressure effect can be modeled explicitly in the Brooks-Corey setting Brooks and Corey (1966). Referring to (5) and since we have written everything in terms of the oil saturation $u=S_{\mathrm{o}}=1-S_{\mathrm{w}}$, the entry pressure is defined by

$$
p_{\mathrm{c}}(x, 0)=\sigma \sqrt{\frac{\phi}{k(x)}} J(0) .
$$

Here and below the dimensionless absolute permeabilities of the coarse and the fine medium are denoted $k^{+}$and $k^{-}$, respectively. Then $k(x)=k^{+}$if $x$ is located in a coarse layer and $k(x)=k^{-}$for $x$ inside a fine layer. Since $k$ is different inside two adjacent homogeneous layers, the entry pressure will be discontinuous at the interface separating the two layers. The situation is different, for example, in the parameterization proposed by van Genuchten (1980). There $p_{\mathrm{c}}(x, 0)=0$, so the entry pressure effect is not present anymore.

In the current setting, due to the non-zero entry pressure, the capillary pressure can only be continuous if both phases are present on each side of the interface. Then both phase pressures are continuous, too. On the other hand, if oil is absent in the fine material, the difference in the entry pressure leads to a discontinuous capillary pressure. Recalling (12), this can be expressed in terms of a threshold saturation (entry point), which has to be exceeded before the non-wetting phase can enter the fine layer. This threshold saturation $u^{*}$ is uniquely defined by

$$
\frac{J\left(u^{*}\right)}{\sqrt{k^{+}}}=\frac{J(0)}{\sqrt{k^{-}}} .
$$

The above discussion is presented in Fig. 2. One can easily see there that the continuity in the capillary pressure can only be achieved if the oil pressure $u$ in the coarse layer exceeds the entry point $u^{*}$.

If the above situation is encountered in a one-dimensional setting, it leads to the so-called trapping effect: the oil flow is blocked at the interface between a coarse and

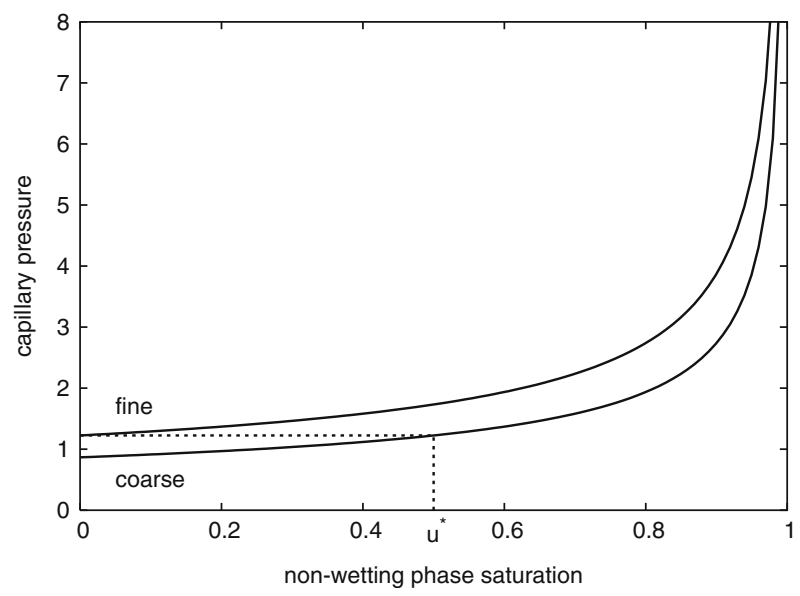

Fig. 2 Entry pressure condition and corresponding saturation $\mathrm{u}^{*}$ 
a fine material. This blocking is encountered as long as the oil saturation at the coarse side of the interface does not exceed the entry point. In the water-driven oil recovery, this means that a certain amount of oil will be trapped at such interfaces. The maximal amount of trapped oil depends on the properties of the layered reservoir and can be determined effectively (see Bertsch et al. 2003; van Duijn et al. 1995, 2002a for details).

In a two- or three-dimensional setting, the entry pressure effect causes a lateral spreading and an eventual bypassing of the less permeable obstacle, since the nonwetting fluid will invade the more permeable media first.

The jumps in the absolute permeability $k$ are inducing discontinuities in the capillary pressure. Therefore the spatial derivatives in (9) and (10) require a special interpretation across the interfaces. To overcome this inconvenience, van Duijn et al. (1995) have proposed to consider Eq. 9 only in the homogeneous layers (where $k$ is constant), and to impose matching conditions across $k$-discontinuities. To be specific, we assume that the interface is located at $x=x_{i}$, and that the highly permeable medium $k^{+}$is located on the left of the interface $\left(x<x_{i}\right)$. Similarly, the fine material $k^{-}\left(k^{+}>k^{-}\right)$is located on the right of the interface $\left(x>x_{i}\right)$. We denote $u\left(x_{i}-, t\right)$ and $u\left(x_{i}+, t\right)$ the values approached by the saturation $u$ at the time $t$ and as coming from the left side and right side of the interface (with the same interpretation for other quantities), respectively.

Further, by

$$
\left[p_{\mathrm{c}}(t)\right]_{x_{i}}=p_{\mathrm{c}}\left(x_{i}+, t\right)-p_{\mathrm{c}}\left(x_{i}-, t\right)
$$

we mean the jump in the pressure at the interface $x_{i}$ and at the time $t$. Then, for all $t>0$, the flux continuity is assumed at $x_{i}$ :

$$
F\left(x_{i}+, t\right)=F\left(x_{i}-, t\right) .
$$

This natural condition is endowed by extended pressure conditions:

$$
u\left(x_{i}+, t\right)\left[p_{\mathrm{c}}(t)\right]_{x_{i}}=0, \text { and }\left[p_{\mathrm{c}}(t)\right]_{x_{i}} \geq 0 .
$$

As follows from the above equality, if oil is present on both sides of the interface $\left(u\left(x_{i}+, t\right)>0\right)$, the capillary pressure must be continuous $\left(\left[p_{\mathrm{c}}(t)\right]_{x_{i}}=0\right)$. On the other hand, if the capillary pressure is discontinuous across the interface $\left(\left[p_{\mathrm{c}}(t)\right]_{x_{i}} \neq 0\right)$, there is no oil on the fine material side of the interface $\left(u\left(x_{i}+, t\right)=0\right)$. Moreover, the capillary pressure has then a positive jump $\left(\left[p_{\mathrm{c}}(t)\right]_{x_{i}} \geq 0\right)$, since on the coarse material side it stays below the entry point. With reference to Fig. 2, the pressure condition (18) can be formulated as

$$
\left\{\begin{array}{l}
u\left(x_{i}-, t\right)<u^{*} \text { implies } u\left(x_{i}+, t\right)=0 \\
u\left(x_{i}-, t\right) \geq u^{*} \text { implies } \frac{J\left(u\left(x_{i}-, t\right)\right)}{\sqrt{k^{+}}}=\frac{J\left(u\left(x_{i}+, t\right)\right)}{\sqrt{k^{-}}},
\end{array}\right.
$$

with $u^{*}$ being the entry point defined in (15).

For a simplified setting, a rigorous mathematical justification of the above procedure has been given by Bertsch et al. (2003).

\subsection{Scale effects}

The entry pressure effect described in the previous subsection is one of the important features that need to be accounted for in the upscaling procedure. It occurs at each of 
the interfaces separating two homogeneous layers having different absolute permeabilities. In mathematical terms, this translates into the matching conditions (17) and (19), which have to be fulfilled at the microscale across any interface.

Our aim here is a rational derivation of an effective equation describing the two-phase flow at the macroscale. To do so, in Sect. 3 we employ homogenization techniques. The resulting model will include the microscale entry pressure effect. In particular, as mentioned in Sect. 2.2, local trapping effects can occur due to the interface conditions. Specifically, the non-wetting phase (oil) cannot enter a less permeable (fine) zone until a certain threshold saturation at the interface is exceeded. This leads to a maximal amount of oil that can be trapped inside a coarse layer. Further, once the maximal amount of trapped oil is exceeded in the coarse material, the amount of oil will not sink below that value, unless other kinds of phenomena occur: obstacle bypassing in the multi-dimensional case, or dissolution and degradation effects. Here we disregard these cases.

On the macroscale, the local trapping phenomena lead to a macroscopic residual saturation. This trapped mass of non-wetting phase becomes immobile and cannot be removed by the flow processes. These assertions are sustained by the analysis carried out in Sect. 3.

\section{The homogenized model}

In this section, we proceed with the derivation of the effective two-phase flow equations. The microscale trapping effects that are due to the differences in the entry pressure corresponding to different homogeneous layers are also included.

\subsection{The homogenization procedure}

To end up with an effective model we apply homogenization techniques (Hornung, 1997). We refer to the setting of Sect. 2.1. The heterogeneity enters into the system only through the alternating high $\left(k^{+}\right)$and low $\left(k^{-}\right)$absolute permeabilities, which are repeating as indicated in Fig. 3. Each homogeneous layer has a constant thickness $L_{y}$ (the microscale reference length), which is much smaller than the macroscale reference length $L_{x}$. This leads naturally to a small expansion parameter $\varepsilon=L_{y} / L_{x}$. Trapping occurs at all transitions from a coarse to a fine layer, as mentioned in Sect. 2.2.

A similar approach can be applied to derive effective equations for a different distribution of the homogeneous layers (see van Duijn et al. 2002a). This includes

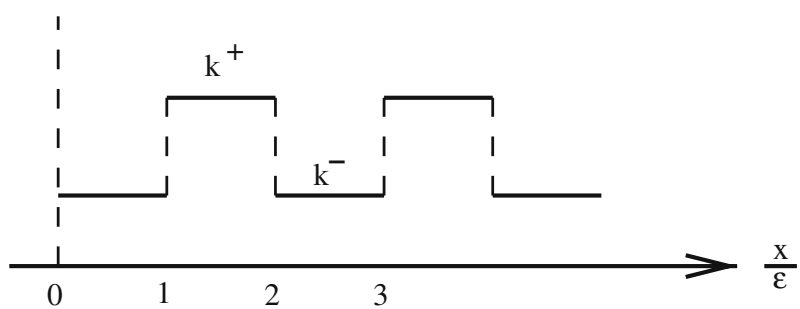

Fig. 3 Permeability distribution on the small scale 
microstructures that are randomly distributed with respect to both the location of the permeability jumps and the value of the permeability.

At this point we should recall the presence of the two dimensionless numbers, $M$ and $N_{\mathrm{c}}$. In van Duijn et al. (2002a) the viscosity ratio is assumed of order 1 , and the cases of moderate and small capillary number $N_{\mathrm{c}}$ are treated separately. While the results obtained there for the balance case $\left(N_{\mathrm{c}}=O(\varepsilon)\right)$ were confirmed numerically by a clear convergence of the averaged solution of the full (microscale) problem towards the effective solution, in the capillary limit case $\left(N_{\mathrm{c}}=O(1)\right)$ this numerical evidence was difficult to achieve unless capillary effects are becoming dominant. This drawback is caused by a microscale assumption concerning the continuity of the $O(\varepsilon)$ term in the asymptotic expansion of the oil saturation. In the present work we focus on the capillary limit case and derive a macroscale equation that is suitable also for the flow regimes where capillary effects are not dominating. Here we make a different microscale assumption: the continuity of the $O(\varepsilon)$ term in the expansion of the capillary pressure. In neither case have we a rigorous mathematical proof for convergence, therefore we restrict ourselves to compare the numerical results. As revealed by the experiments in Sect. 4, which are performed for a moderate capillary effects $N_{\mathrm{c}}=1$, the present approach yields an effective solution that agrees well with the averaged solution of the microscale model. In a similar context, a first convergence result for the homogenization procedure is proven in Schweizer (2006). The oil saturation is assumed there strictly positive. By this assumption, trapping is ruled out since it can only appear whenever the soil saturation $u$ vanishes in the fine material. The additional assumption considered in Schweizer (2006) involves the averaged slopes for $u$ in the adjacent homogeneous layers, leading to an upscaled model that is equivalent to the one derived here.

Now we can proceed with the derivation of the effective (upscaled, or averaged) equations for the limit $\varepsilon \searrow 0$. In doing so we apply homogenization techniques. Specifically, we assume that all the quantities depend on two spatial variables, $x$ being the macroscale (slow) variable, and $y=\frac{x}{\varepsilon}$ the microscale (fast) variable. Having this assumption in mind we expand all the involved quantities asymptotically in $\varepsilon$ and equate the terms of the same order in $\varepsilon$. For example, the multiscale oil saturation $u_{\varepsilon}$ is expanded as

$$
u_{\varepsilon}(x, t)=u^{0}(x, y, t)+\varepsilon u^{1}(x, y, t)+\varepsilon^{2} u^{2}(x, y, t)+\cdots
$$

where the functions $u^{j}$ are periodic in $y=x / \varepsilon$. In this article, we will only point out the differences between the two above mentioned assumptions for the effective equations. For all the steps necessary in the derivation we refer to van Duijn et al. (2002a).

At the interface between the two layers the fluxes on all scales are continuous. Moreover, the pressure condition (19) is imposed at $y=0$, and periodicity is assumed at the end points of the cells. These conditions can only be fulfilled if $u^{0}$ the lowest order term in the expansion 20, does not depend on $y$ in any of the homogeneous layers. To be specific, with $c$ and $\bar{c}$ related by

$$
\frac{J(c)}{\sqrt{k^{+}}}=\frac{J(\bar{c})}{\sqrt{k^{-}}}
$$

whenever $\bar{c}>0$, we have

Springer 


$$
u^{0}(y)= \begin{cases}c>u^{*} & \text { for } \quad-1<y<0 \\ \bar{c} & \text { for } \quad 0<y<1\end{cases}
$$

if $c$ exceeds the threshold saturation $u^{*}$ and the NAPL can therefore infiltrate into the fine material, or

$$
u^{0}(y)= \begin{cases}c \leq u^{*} & \text { for } \quad-1<y<0 \\ 0 & \text { for } \quad 0<y<1\end{cases}
$$

if there is no NAPL flow from the coarse into the fine material. In the first case, the capillary pressure is continuous at the interface. In contrast to the derivation of van Duijn et al. (2002a), who are assuming that $u^{1}$ is continuous at the interface between coarse and fine sand, we propose a different approach: continuity is assumed for $p_{\mathrm{c}}^{1}$, the first-order term in the expansion of the capillary pressure. Even though both assumptions are made on a $O(\varepsilon)$ scale, the resulting macroscale models are different. The interface conditions for $u^{1}$ become in our case.

To proceed with the derivation of the upscaled model, we first notice that the capillary pressure can be expanded asymptotically as

$$
\begin{aligned}
p_{\mathrm{c}}\left(u_{\varepsilon}\right) & =\frac{J\left(u_{\varepsilon}\right)}{\sqrt{K}} \\
& =\frac{1}{\sqrt{K}}\left(J\left(u^{0}\right)+\left.\frac{\partial J(u)}{\partial u}\right|_{u^{0}} \Delta u+\left.\frac{\partial^{2} J(u)}{(\partial u)^{2}}\right|_{u^{0}}(\Delta u)^{2}+\cdots\right)
\end{aligned}
$$

with $\Delta u=\varepsilon u^{1}+\varepsilon^{2} u^{2}+\cdots$.

This gives

$$
p_{\mathrm{c}}\left(u_{\varepsilon}\right)=\frac{1}{\sqrt{K}}\left(J\left(u^{0}\right)+\varepsilon u^{1} J^{\prime}\left(u^{0}\right)+\cdots\right),
$$

the dots standing for terms of order $\varepsilon^{2}$.

The first two terms in the $p_{\mathrm{c}}$-expansion are:

$$
\begin{gathered}
p_{\mathrm{c}}^{0}=\frac{J\left(u^{0}\right)}{\sqrt{K}} \\
p_{\mathrm{c}}^{1}=\frac{u^{1} J^{\prime}\left(u^{0}\right)}{\sqrt{K}} .
\end{gathered}
$$

Assuming that $p_{\mathrm{c}}^{1}$ is continuous in $y=0$ and at the end points $y= \pm 1$. The interface conditions for $u^{1}$ become

$$
\begin{gathered}
u^{1}(0-) \frac{J^{\prime}(c)}{\sqrt{k^{+}}}=u^{1}(0+) \frac{J^{\prime}(\bar{c})}{\sqrt{k^{-}}}, \\
u^{1}(-1+) \frac{J^{\prime}(c)}{\sqrt{k^{+}}}=u^{1}(1-) \frac{J^{\prime}(\bar{c})}{\sqrt{k^{-}}} .
\end{gathered}
$$


3.2 The effective equation

With

$$
U(x, t)=\frac{1}{2} \int_{-1}^{1} u^{0}(x, y, t) \mathrm{d} y=\frac{1}{2}(c+\bar{c}),
$$

the effective equation has the following form:

$$
\partial_{t} U+\partial_{x}\left[\mathcal{F}(U)-N_{\mathrm{c}} \Lambda(U) \partial_{x} \mathcal{P}_{C}(U)\right]=0 .
$$

The effective saturation is expressed in terms of $c$ and $\bar{c}$. For $\bar{c}>0$, using (21) we end up with

$$
\bar{c}=J^{-1}\left(\sqrt{k^{-} / k^{+}} J(c)\right) .
$$

This gives

$$
U=G(c):=\frac{1}{2}\left(c+J^{-1}\left(\sqrt{k^{-} / k^{+}} J(c)\right)\right) .
$$

Notice that $G$ defined above maps $(0,1)$ onto $\left(\frac{1}{2} u^{*}, 1\right)$. Since $J$ is strictly increasing, the function $G$ shares the same property and can therefore be inverted. So we can write $c$ in terms of $U$ :

$$
c=G^{-1}(U)
$$

Inserting the above into (32) we obtain an equivalent expression for $\bar{c}$.

With the above expressions we can identify the upscaled fractional flow:

$$
\mathcal{F}(U)= \begin{cases}0 & \text { for } \quad 0 \leq U \leq \frac{1}{2} u^{*}, \\ \frac{\frac{1}{k^{+} k_{w}(c)}+\frac{1}{k^{-} k_{w}(\bar{c})}}{\frac{1}{k^{+} k_{w}(c) f(c)}+\frac{1}{k^{-} k_{w}(\bar{c}) f(\bar{c})}} & \text { for } \quad \frac{1}{2} u^{*}<U<1, \\ 1 & \text { for } \quad U=1 .\end{cases}
$$

This function can be interpreted as the weighted harmonic mean of the microscale fractional flow. The weights, $1 /\left(k^{+} k_{\mathrm{W}}(c)\right)$ and $1 /\left(k^{-} k_{\mathrm{W}}(\bar{c})\right)$, are depending on the relative water permeabilities, which, in turn are depending on $c$ and $\bar{c}$.

The upscaled $\Lambda$ term is the harmonic average of the terms $k^{+} \lambda(c)$ and $k^{-} \lambda(\bar{c})$,

$$
\Lambda(U)=\left\{\begin{array}{lll}
0 & \text { for } \quad 0 \leq U \leq \frac{1}{2} u^{*}, \\
\frac{2}{\frac{1}{k^{+} \lambda(c)}+\frac{1}{k^{-\lambda}(\bar{c})}} & \text { for } \quad \frac{1}{2} u^{*}<U<1, \\
0 & \text { for } \quad U=1 .
\end{array}\right.
$$

To express the upscaled capillary pressure we recall (21). This means that, whenever oil is present on both sides of the interface, the first term in the expansion (25) is constant in the entire microscale cell. Therefore we get

$$
\mathcal{P}_{\mathrm{c}}(U)=\frac{J(c)}{\sqrt{k^{+}}}=\frac{J(\bar{c})}{\sqrt{k^{-}}} .
$$


To determine the effective diffusivity $\mathcal{D}$ we use (34), (36), and (37), yielding

$$
\mathcal{D}(U)=\Lambda(U) \mathcal{P}_{\mathrm{c}}^{\prime}(U), \quad \text { where } \quad \mathcal{P}_{\mathrm{c}}^{\prime}(U)=\frac{1}{\sqrt{k^{+}}} \frac{J^{\prime}\left(G^{-1}(U)\right)}{G^{\prime}\left(G^{-1}(U)\right)}
$$

Remark. In van Duijn et al. (2002a) the effective equation was derived assuming the continuity of $u^{1}$ across the interfaces separating the fine and coarse layers. This yields discontinuities of order $\varepsilon$ in the capillary pressure. Though small, these discontinuities result in a noticeable effect at the macroscale. In fact, this observation motivated us to write this article. Without a rigorous mathematical convergence proof one relies on numerical simulations to justify the underlying assumptions. As shown in Section 4 , the assumption of continuity of $p_{c}^{1}$ yields better results. In van Duijn et al. (2002a) the first non-zero term in the expansion of the flux reads

$$
F^{1}=\frac{\frac{f(C)}{\sqrt{k^{+}} D(C)}+\frac{f(\bar{C})}{\sqrt{k^{-}} D(\bar{C})}}{\frac{1}{\sqrt{k^{+}} D(C)}+\frac{1}{\sqrt{k^{-}} D(\bar{C})}}-N_{\mathrm{c}} \frac{\frac{\partial C}{\partial x}+\frac{\partial \bar{C}}{\partial x}}{\frac{1}{\sqrt{k^{+}} D(C)}+\frac{1}{\sqrt{k^{-}} D(\bar{C})}} .
$$

By the procedure described above we end up with an effective equation that is similar to (31). In both approaches, the effective functions vanish for $0 \leq U \leq 1 / 2 u^{*}$, yielding the same maximal amount of trapped oil. The differences occur in the nontrivial case, when $1 / 2 u^{*}<U<1$. In both upscaled equations, the effective fractional flow is a weighted harmonic mean of the microscale fractional flow. The difference is in the form of the weights. A straightforward calculation shows that the weights in (39) are $1 /\left(\sqrt{k^{+}} k_{\mathrm{W}}(c) J^{\prime}(c)\right)$ and $1 /\left(\sqrt{k^{-}} k_{\mathrm{w}}(\bar{c}) J^{\prime}(\bar{c})\right)$. These are different from the ones appearing in (35). Further, assuming $u^{1}$ continuous gives directly an effective diffusivity, which is the harmonic mean of the microscale diffusivities. In (31) we first determine the effective $\Lambda$ function and next $\mathcal{D}$ according to (38). The differences are illustrated in the next section for the example considered there, see Fig. 4.
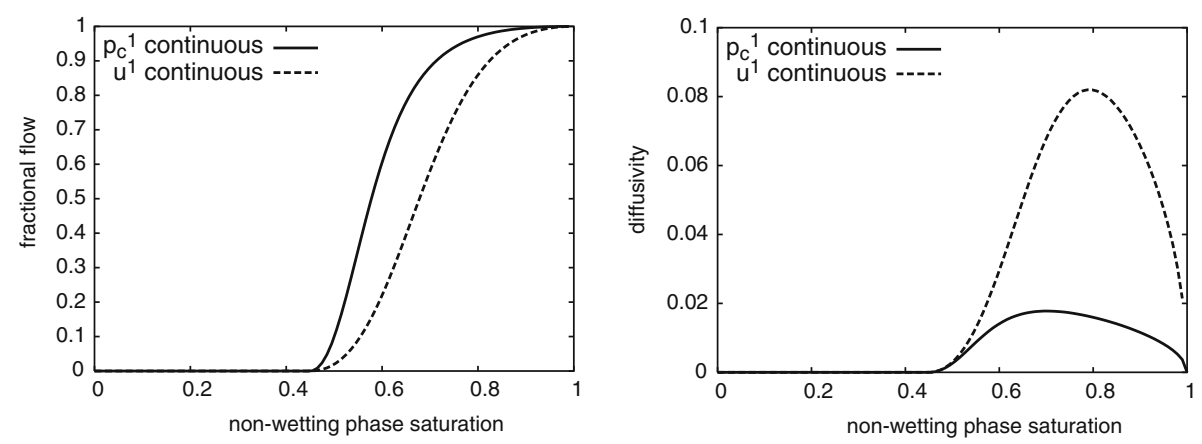

Fig. 4 The effective fractional flows (left) and diffusivities (right) obtained for the present approach (solid), respectively the approach in van Duijn et al. (2002a) (dashed). Here $M=1$ and $k^{+} / k^{-}=10$ 


\section{Comparison of upscaled and small-scale model}

In this section, we first compare the two different upscaled models and then compare the small-scale numerical simulations with our upscaled model equations. For this we use the numerical simulator MUFTE-UG (Bastian and Helmig, 1999) with a control volume finite element implicit scheme to solve the two-phase flow equations on the small scale and a first-order explicit discretization scheme with upwind finite volumes for the upscaled one-dimensional model.

The one-dimensional code solves directly the saturation equation (31). For the simulation on the small scale performed in MUFTE-UG we use a saturation-pressure formulation for which appropriate initial and boundary conditions have to be added. To be specific, initially the whole domain is in both cases fully oil saturated $(u=1)$ except for one special case, where we only have one blob of oil in our domain. For the effective equation we set $u=0$ and $u=1$ at the left and the right boundary, respectively. The same is considered for the saturation equation at the microscale. In addition, the boundary conditions for the pressure equation are chosen such that the total flux remains constant.

The capillary number $N_{\mathrm{c}}$ is set to 1 . The dimensionless interval of computation is $(0,2)$, yielding $L_{x}=2$. We use Brooks-Corey type relative permeabilities and Leverett type capillary pressure:

$$
k_{\mathrm{rw}}(u)=(1-u)^{2}, \quad k_{\mathrm{ro}}(u)=u^{2}, \quad J(u)=(1-u)^{-0.5} .
$$

The entry point depends only on the two different sands. For $k^{+} / k^{-}=10$ and $k^{+} / k^{-}=$ 2 the entry points are $u^{*}=0.9$ and $u^{*}=0.5$, respectively. This results in $U^{*}=0.45$ in the former case and $U^{*}=0.25$ in the latter. Diffusion is not dominating, and the effective model obtained assuming $u^{1}$ continuous was not in good agreement with the averaged small-scale solution (see van Duijn et al. 2002b). The effective fractional flows and diffusivities, as computed in this setting for both the approach in van Duijn et al. (2002a) and the present one, are compared in Fig. 4 for $k^{+} / k^{-}=10$. Notice the enhanced diffusive effects in the former case. One can clearly see that in both cases the phases are immobile until the upscaled threshold saturation $U^{*}$ has been exceeded.

The corresponding effective solutions, computed at $t=0.5$, are compared in Fig. 5. Obviously, the two solutions are not identical. The solution profile is steeper in the present approach, which is a direct consequence of the difference in the effective diffusion. Further as suggested by the differences in the effective fractional flow functions, the solution fronts in the two cases should have different velocities.

Having in mind the above, it is natural to ask ourselves which of the two effective models is appropriate. In the absence of a rigorous mathematical proof, our choice is dictated by the numerical evidence. To this aim we solve the full problem at the microscale, with the domain $(0,2)$ consisting of $N$ cells comprising a coarse and a fine layer.

In our example we consider four different cases, as described in Table 1.

In the first case computations are performed for $N$ being 20,40, or 80 . This gives $\varepsilon=1 / 20,1 / 40$, or $1 / 80$, and $L_{y}=\varepsilon L_{x} / 2$. For comparison with the effective solution, the computed small-scale solution is averaged over each microscale cell. As $\varepsilon \searrow 0$, the averaged solution converges towards the effective one. The numerical evidence for convergence is given in Fig. 6. The differences on the left side are explained by the fact that the first average point is located further into the domain, this effect being 


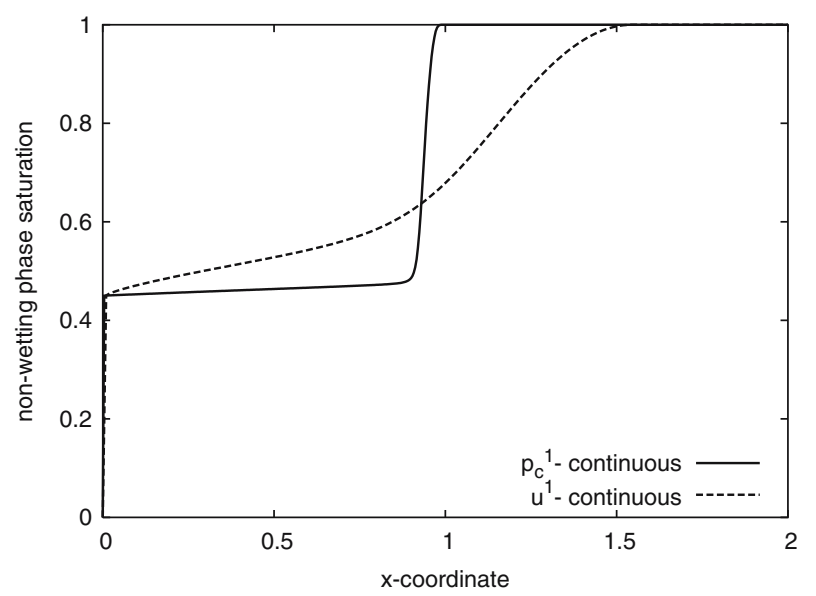

Fig. 5 Effective solutions, obtained at $t=0.8$ for the present approach (solid) and the approach in van Duijn et al. (2002a) (dashed). Here $M=1$ and $k^{+} / k^{-}=10$

Table 1 Parameters and initial conditions used in the test cases

\begin{tabular}{lcll}
\hline Case \# & $k^{+} / k^{-}$ & $M$ & Initial condition \\
\hline 1 & 2 & 1 & Fully oil saturated \\
2 & 10 & 1 & Fully oil saturated \\
3 & 2 & 5 & Fully oil saturated \\
4 & 2 & 1 & Blob of oil \\
\hline
\end{tabular}

more obvious for larger values of $\varepsilon$. In the flat region, the curves for all values of $\varepsilon$ are on top of each other. As $\varepsilon \searrow 0$, the convergence is improved also in the front region.

Figure 7 shows the solution on the small scale for $\varepsilon=1 / 80$ together with its cellaverage. Notice that the jumps at the interface are higher in regions where $u$ is closer to zero. This is due to (21). The differences are getting smaller as the non-wetting phase saturation increases.

As we can also see in the right picture in Fig. 7, the solution inside each layer is not constant. This contradicts (22), used in the derivation of the effective model, and meaning that the first term in the asymptotic expansion of the saturation, $u_{0}$ is not layer-wise constant. This difference can be explained by the fact that the computed solution incorporates also $O(\varepsilon)$ effects. These effects will vanish as $\varepsilon \searrow 0$, yielding piecewise constant solutions.

The comparison of the effective and the averaged small-scale solution for different times is shown in Fig. 8. It can be seen that in both cases, since we start our numerical experiments with a fully oil saturated domain, the saturation does not drop below the macroscopic residual saturation $U^{*}$. This value represents the maximal amount of oil that will be trapped due to small-scale heterogeneities. On the small scale, this is caused by the fact that there is no oil flow from a coarse layer into a fine one, unless the oil saturation in the coarse layer exceeds the entry point $u^{*}$. At the macroscale, this translates into effective convection and diffusion vanishing on the interval $\left[0, U^{*}\right]$.

One can also notice a slightly less good agreement in the region where the solution is close to $U^{*}$. This can be explained by the higher amplitude in the oscillations, 


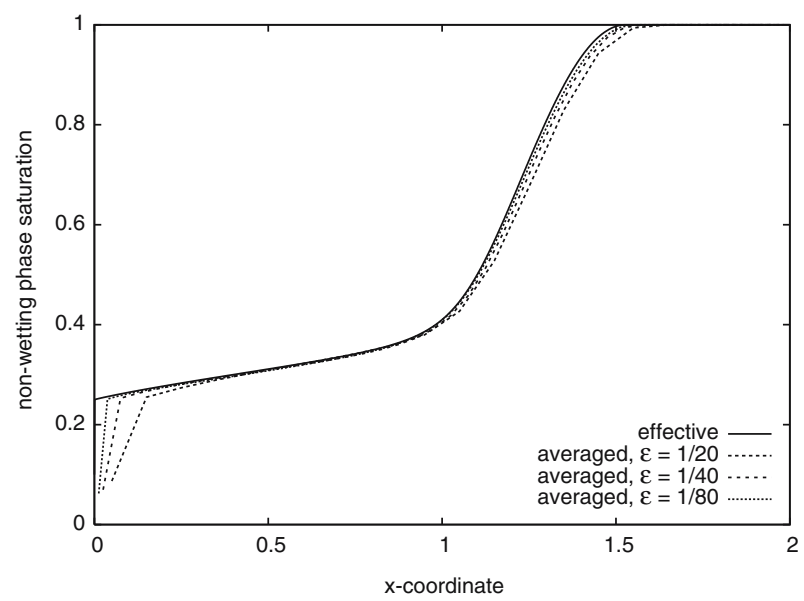

Fig. 6 Effective saturation (solid), computed assuming $p_{\mathrm{c}}^{1}-$ continuous, and averaged saturations at $t=0.8$
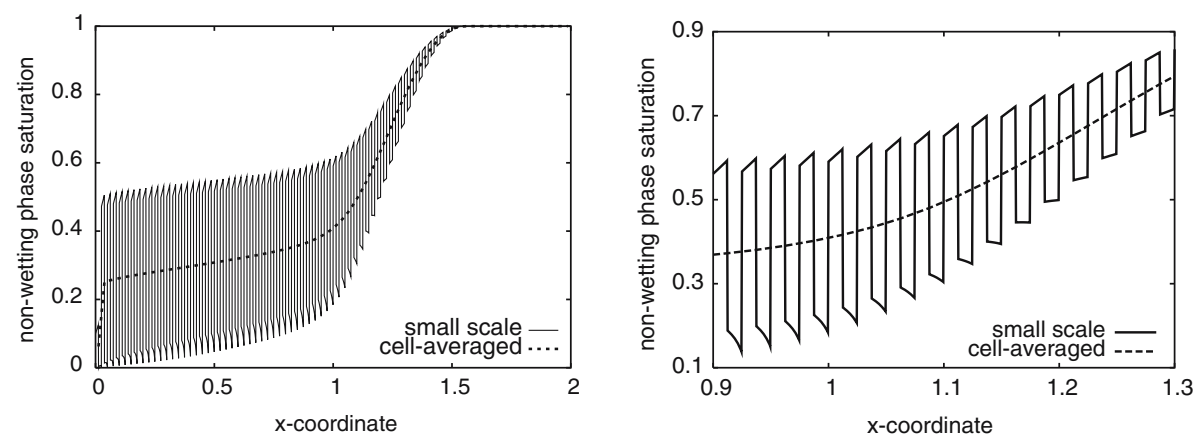

Fig. 7 Small-scale problem: oscillatory (solid) and averaged (dashed) oil saturation at $t=0.8$, full (left) and zoomed view (right) for $\varepsilon=1 / 80$

encountered-as mentioned above - for such values of the averaged solution. But taking into account also the front region, as well as its velocity, we conclude that the two solutions are matching well in both qualitative and quantitative sense. In consequence, we consider the effective model derived assuming the continuity of $p_{\mathrm{c}}^{1}$ to be more appropriate than the one in van Duijn et al. (2002a), in particular for the flow regimes that are not dominated by diffusion.

The model is not restricted to small permeability contrasts and can also handle differences in viscosity. By incorporating these effects we have to keep in mind that the expansion of the variables is based on the parameter $\varepsilon$ alone. It is therefore only valid if $\varepsilon$ is much smaller than any of the other parameters contrasts. Assuming that the oscillations in the parameters involved in the micromodel are also $\varepsilon$ dependent, we would end up with different upscaled models. However, their study is beyond the scope of the present investigation.

As mentioned in Table 1 we consider three further cases in which always one parameter is different compared to the first case: a higher permeability contrast $k^{+} / k^{-}=10$, 


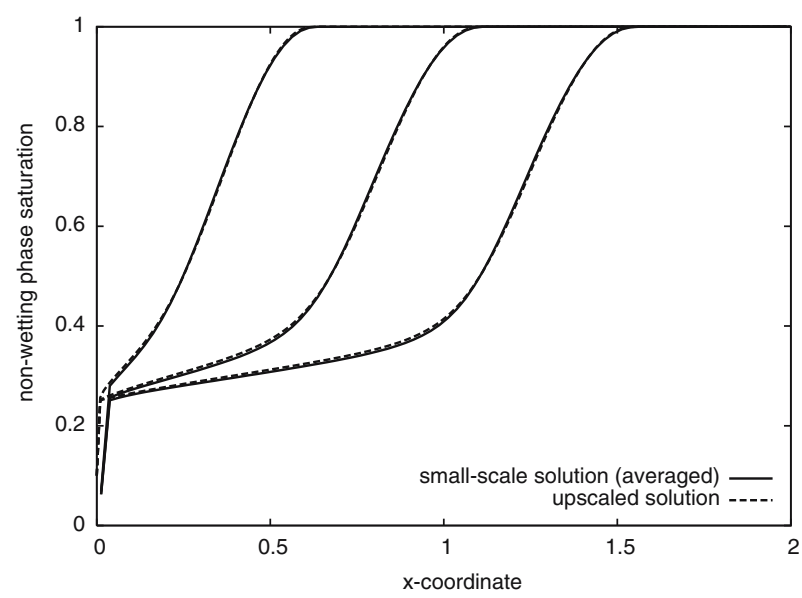

Fig. 8 Case 1: upscaled and averaged solution, $t=0.2,0.5$, and 0.8 for $\varepsilon=1 / 80$

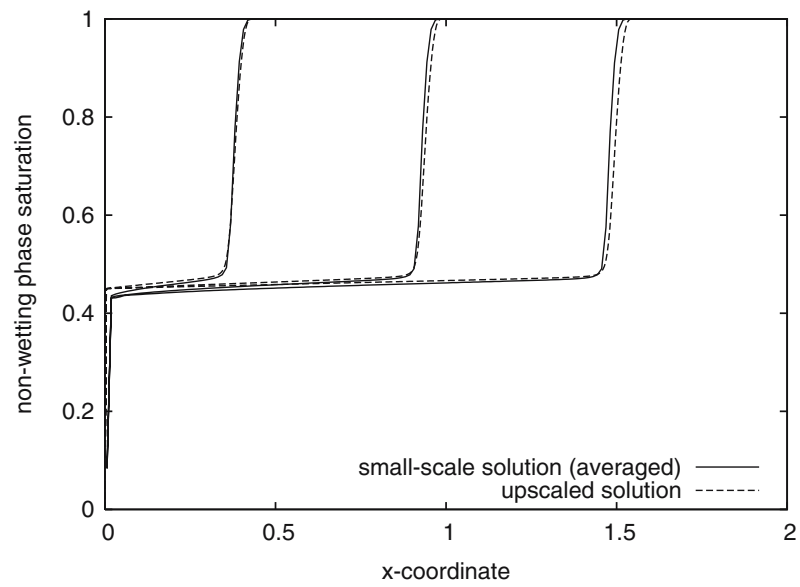

Fig. 9 Case 2: upscaled and averaged small-scale results after $t=0.2,0.5$ and 0.8 for $\varepsilon=1 / 80$

a different viscosity ratio $M=5$, and a different initial condition, where only a part of the domain is saturated.

First, we evaluate a higher permeability ratio of $k^{+} / k^{-}=10$. Since we make use of the Leverett model (5), this leads directly to a higher difference between the two entry pressures, yielding an entry point of $u^{*}=0.9$, and therefore $U^{*}=0.45$ as can be seen in Fig. 9.

The shape of the curve is now almost angular. The saturation is nearly constant from the left boundary until directly behind the front, where the saturation jumps to the initial saturation. The two different solutions match very well, only the front velocity is a little overestimated by the upscaled solution. As mentioned above, this can also be explained by the model assumption that the saturation is constant in each layer, which only holds in the limit case $\varepsilon \searrow 0$.

The viscosity ratio $M$ is another important parameter that influences the flow pattern. $M$ appears in the denominator of the fractional flow function (11), larger 


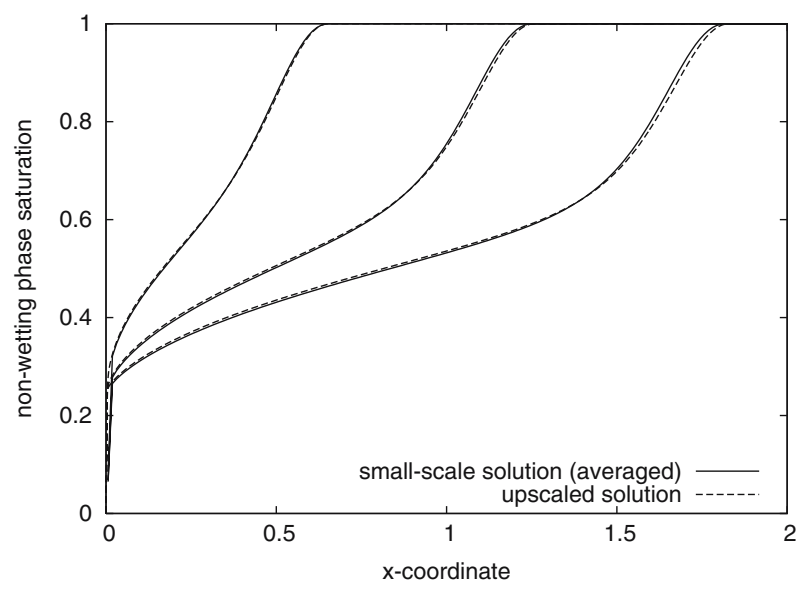

Fig. 10 Case 3: upscaled and averaged small-scale results after $t=0.2,0.5$ and 0.8 for $\varepsilon=1 / 80$

values of $M$ therefore shift the balance between viscous and capillary forces in the direction of the capillary forces. As a direct consequence, the front velocity is changing as well.

In Fig. 10, the non-wetting phase saturation is plotted over the x-coordinate at three points in time. The solid curves represent the cell-averaged small-scale results, while the dashed curves show the results obtained with our upscaled equation. The two different curves lie almost on top of each other. The upscaled solution overestimates the front velocity slightly, which can be seen for $t=0.8$. In comparison to Fig. 8 one can observe the influence of the viscosity ratio. For $M=5$ the diffusive influence gets larger, i.e., the flat part of the curve gets steeper and vice versa. Furthermore, the front is also smeared out.

In the last set-up we change our initial conditions. The major part of the domain is filled with water and a blob of oil with a saturation of $u=0.9$ is placed in the interval $0.5<x<1.0$.

In Fig. 11, we see the temporal evolution of the oil saturation. Due to the flow from left to right, the oil is mainly displaced to the right. Only a small amount is transported against the direction of flow. The left side of the curve is not exactly matched but in fairly good agreement, while on the right front we have an almost perfect match.

\section{Final remarks}

We have derived an upscaled equation for a periodically layered medium. In doing so we have employed the method of homogenization. Trapping effects that appear on the small scale due to the difference in the entry pressure corresponding to a homogeneous layer are explicitly taken into account. The resulting effective model is a non-linear parabolic problem of degenerate type, incorporating convection. The effective functions can be seen as weighted harmonic means of the corresponding small scale ones. These functions vanish whenever the effective saturation $U$ is below $U^{*}$, representing the maximal amount of trapped oil. 


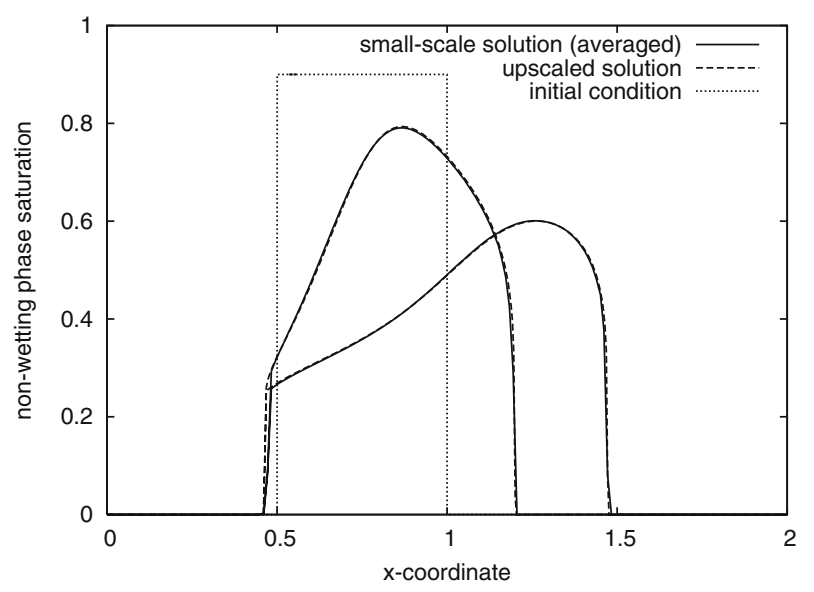

Fig. 11 Case 4: upscaled and averaged small-scale results after $t=0.1$ and 0.3 for $\varepsilon=1 / 80$

It has to be stressed that these results are only valid for moderate capillary numbers $\left(N_{\mathrm{c}}=O(1)\right)$. For different regimes, i.e., $N_{\mathrm{c}}=O\left(\varepsilon^{\alpha}\right)$, the homogenization approach will lead to equations of different type (see van Duijn et al. 2002a).

Acknowledgements We would like to thank Dr. Insa Neuweiler for bringing to our attention the effect that is associated with the continuity assumption on the microscale. This research was supported through a European Community Marie Curie Fellowship (Contract number HPMT-CT-200100422) while H. Eichel spent 6 months at the TU Eindhoven. Also, this work has been supported within the DFG-project FIMOTUM under the grant He 2531/5-1, and by the Dutch government through the national program BSIK: knowledge and research capacity, in the ICT project BRICKS (http://www.bsik-bricks.nl), theme MSV1.

\section{References}

Bastian, P., Helmig R.: Efficient fully-coupled solution techniques for two-phase flow in porous media. Adv. Water Resour. 23, 199-216 (1999)

Bertsch, M., Dal Passo, R., van Duijn, C.J.: Analysis of oil trapping in porous media flow. SIAM J. Math. Anal. 35, 245-267 (2003)

Brooks, R.H., Corey, A.T.: Properties of porous media affecting fluid flow. J. Irrig. Drain. Div. Proc. ASCE 92, 61-88 (1966)

Darman, N.H., Pickup, G.E., Sorbie, K.S.: A comparison of two-phase flow dynamic upscaling methods based on fluid potentials. Comp. Geosci. 6, 5-27 (2002)

van Duijn, C.J., Mikelic, A., Pop, I.S.: Effective equations for two-phase flow with trapping on the micro scale. SIAM J. Appl. Math. 62(5), 1531-1568 (2002a)

van Duijn, C.J., Mikelic, A., Pop, I.S.: Effective Buckley-Leverett equations by homogenization. In: Anile, M., Capasso, V., Greco, A. (eds.) Progress in Industrial Mathematics at ECMI 2000: Mathematics in Industry, vol. 1, pp. 42-51. Springer-Verlag, Heidelberg (2002b)

van Duijn, C.J., Molenaar, J., de Neef, M.J.: The effect of capillary forces on immiscible two-phase flow in heterogeneous porous media. Transp. Porous Media 21, 71-93 (1995)

van Genuchten, M.Th.: A closed-form equation for predicting the hydraulic conductivity of unsaturated soils. Soil Sci. Soc. Am. J. 44, 892-898 (1980)

Helmig, R.: Multiphase Flow and Transport Processes in the Subsurface. Springer-Verlag, Heidelberg (1997) 
Hornung, U.: Homogenization and Porous Media. Interdisciplinary Applied Mathematics, vol. 6. Springer Verlag, New York (1997)

Illangasekare, T.H., Ramsay, J.L., Jensen, K.H., Butts, M.B.: Experimental study of movement and distribution of dense organic contaminants in heterogeneous aquifers. J. Contam. Hydrol. 20(1-2), $1-25$ (1995)

Kueper, B.H., Abbott, W., Farquhar, G.: Experimental observations of multiphase flow in heterogeneous porous media. J. Contam. Hydrol. 5, 83-95 (1989)

Kueper, B.H., Frind, E.O.: Two-phase flow in heterogeneous porous media. 1. Model development. Water Resour. Res. 27, 1049-1057 (1991)

Leverett, M.C.: Capillary behavior in porous solids. Trans. AIME Petr. Eng. Div. 142, 152-169 (1941)

Lewandowska, J., Szymkiewicz, A., Burzyński, K., Vauclin, M.: Modeling of unsaturated water flow in double porosity soils by the homogenization approach. Adv. Water Resour. 27(3), 321-345 (2004)

van Lingen, P.: Quantification and reduction of capillary entrapment in cross-laminated oil reservoirs, PhD Thesis. Sub-Faculty of Applied Earth Sciences, Delft University of Technology (1998)

Neuweiler, I., Cirpka, O.A.: Homogenization of Richards equation in permeability fields with different connectivities. Water Resour. Res. 41, W02009, doi:10.1029/2004WR003329 (2005)

Schweizer, B.: Homogenization of degenerate two-phase flow equations with oil-trapping. Preprint 2006-04, Department of Mathematics, University of Basel (submitted) (2006)

Wen, X.H., Gómez-Hernández, J.J.: Upscaling hydraulic conductivities in heterogeneous media: an overview. J. Hydrol. 183, ix-xxxii (1996) 